\title{
COMPOSITION CONDITIONS FOR TWO-DIMENSIONAL POLYNOMIAL SYSTEMS
}

\begin{abstract}
M.A.M. ALWASH
Abstract. Several papers published recently about the center composition condition of Abel differential equations and two-dimensional polynomial systems with homogeneous nonlinearities. We give necessary and sufficient conditions for the existence of composition centers for general two-dimensional polynomial systems.
\end{abstract}

Mathematics subject classification (2010): 34C07, 34C05, 34C25, 37G15.

Keywords and phrases: two-dimensional polynomial systems, center problem, composition condition, Abel differential equation.

\section{REFERENCES}

[1] M.I. Al'muhamedov, On conditions for the existence of stable and unstable centers, Doklady Akademii Nauk SSR(N.S.) 67 (1949), 961-964; Amer. Math. Soc. Trans., no. 34 (1950).

[2] M.A.M. Alwash, The composition conjecture for Abel equation, Expos. Math., 27 (2009), 241-250.

[3] M.A.M. Alwash, On a condition for a center of cubic non-autonomous equations, Proc. Roy. Soc. Edinburgh Sect. A, 113 (1989), 289-291.

[4] M.A.M. Alwash, Computing Poincare-Liapunov constants, Differ. Equ. Dyn. Syst., 6 (1998), 349 361.

[5] M. BLinov, Y. Yomdin, Generalized center conditions and multiplicities for polynomial Abel equations of small degrees, Nonlinearity 12 (1999), 1013-1028.

[6] M. Briskin, J.-P. Francoise, Y. Yomdin, Center conditions, compositions of polynomials and moments of polynomials on algebraic curves, Ergodic Theory Dynam. Systems, 19 (1999), 12011220.

[7] M. BRIS KIn, N. RoYTVARF, Y. Yomdin, Center conditions at infinity for Abel differential equations, Ann. of Math., 172 (2010), 437-483.

[8] A. BRUdnYI, On the center problem of ordinary differential equations, Amer. J. Math., 128 (2006), 419-451.

[9] A. Cima, A. Gasull, F. Manosas, The solution of the composition conjecture for Abel equations, Preprint, gsd.uab.cat (2011).

[10] C. Christopher, Abel equations: composition conjecture and the model problem, Bull. Lond. Math. Soc., 32 (2000), 332-338.

[11] J.-P. Francoise, Parametric centers for trigonometric Abel equations, J. Dynam. Differential Equations, 20 (2008), 777-786.

[12] J. Gine, M. GraU, J. Llibre, Universal centers and composition conditions, Preprint, gad.uab.cat (2011).

[13] F. Pakovich, A counterexample to the composition conjecture, Proc. Amer. Math. Soc., 130 (2002), 3747-3749.

[14] Y. YomDin, The center problem for the Abel differential equation, composition of functions, and moment conditions, Mosc. Math. J., 3, 3 (2003), 1167-1195. 